\title{
Managing myelodysplastic symptoms in elderly patients
}

\author{
This article was published in the following Dove Press journal: \\ Clinical Interventions in Aging \\ 14 October 2009 \\ Number of times this article has been viewed
}

R Ria

M Moschetta

A Reale

G Mangialardi

A Castrovilli

A Vacca

F Dammacco

Department of Biomedical Sciences and Human Oncology, Section of Internal Medicine and Clinical Oncology, University of Bari Medical School, Bari, Italy
Correspondence: Roberto Ria Department of Internal Medicine and Clinical Oncology, University of Bari Medical School, Policlinico - Piazza Giulio Cesare, I I I-70I 24 Bari, Italy

Tel +39-080-547-83-87

Fax +39-080-547-88-59

Email ria@dimo.uniba.it
Abstract: Most patients with myelodysplastic syndromes (MDS) are elderly (median age range 65 to 70 years); as a consequence, the incidence and prevalence of these diseases are rising as the population ages. Physicians are often uncertain about how to identify patients who may benefit from specific treatment strategies. The International Prognostic Scoring System is a widely used tool to assess the risk of transformation to leukemia and to guide treatment decisions, but it fails to take into account many aspects of treating elderly patients, including comorbid illnesses, secondary causes of MDS, prior therapy for MDS, and other age-related health, functional, cognitive, and social problems that affect the outcome and managing of myelodysplastic symptoms. Patients with low-risk disease traditionally have been given only best supportive care, but evidence is increasing that treatment with novel non-conventional drugs such as lenalidomide or methyltransferase inhibitors may influence the natural history of the disease and should be used in conjunction with supportive-care measures. Supportive care of these patients could also be improved in order to enhance their quality of life and functional performance. Elderly patients commonly have multiple medical problems and use medications to deal with these. In addition, they are more likely to have more than one health care provider. These factors all increase the risk of drug interactions and the consequent treatment of toxicities. Manifestations of common toxicities or illnesses may be more subtle in the elderly, owing to age-associated functional deficits in multiple organ systems. Particularly important to the elderly MDS patient is the age-related decline in normal bone marrow function, including the diminished capacity of response to stressors such as infection or myelosuppressive treatments. Through the integration of geriatric and oncological strategies, a personalized approach toward this unique population may be applied. As with many diseases in the elderly, reliance on family members or friends to maintain the prescribed treatments, including travel to and from appointments, may place additional stressors on the patient and his/her support network. Careful evaluation and knowledge of functional status, ability to tolerate treatments, effect of disease progression, and general overall health conditions can provide the best opportunity to support these patients. Immediate assessment of daily living activities may detect deficiencies or deficits that often require early interventions.

Keywords: elderly, myelodysplastic syndromes, novel agents, supportive-care, treatment strategies

\section{Introduction}

Aging is associated with a higher risk of developing malignant diseases in general together with increased vulnerability to other age-related health, functional, and social problems. The majority of patients with myelodysplastic syndromes (MDS) are older than 55 years of age. Aging is an important risk factor for the development 
of these diseases. Uncertainty persists about how to identify patients who would benefit most from intensive curative treatment such as hematopoietic stem cell transplantation (HSCT) or from no or low-intensity treatment with growth factors, immunomodulating agents, or low-intensity chemotherapy for managing disease symptoms. Some patients may do best with supportive care alone or may not be candidates for any treatment at all. As the proportion of older persons in the population increases, it will become extremely important to identify which older patients are most suitable for different types of therapy.

\section{Epidemiology}

MDS comprise a heterogenous group of disorders which are the most common hematologic diseases in elderly patients. The incidence of MDS from 2001 to 2003 was 3.3 per $100,000 .{ }^{1}$ The overall 3 -year survival rate was $45 \%$; lower for males than for females. In 2004 it was estimated that there were about 9,700 patients with MDS in the United States, a number that may underestimate its prevalence. MDS incidence rates significantly increased with each calendar year from 2001 to 2004 and only $4 \%$ of patients were reported to registries by physicians' offices. ${ }^{1}$ Data to support a higher incidence of MDS in the United States can be inferred from the third National Health and Nutrition Examination Survey (NHANES III). ${ }^{2}$ These show that the prevalence of anemia rose sharply in adults over 50 , exceeding $20 \%$ for those aged 85 and older. Overall, $11 \%$ of men and $10.2 \%$ of women 65 -years or older were anemic. One-third of those with anemia presented evidence of nutrient deficiency, another third presented anemia of chronic disease and/or renal insufficiency, and those remaining were diagnosed with unexplained anemia. MDS is likely to be a more precise diagnosis for many patients classified as having unexplained anemia. Among them, $17.2 \%$ (5.8\% of the total population with anemia) had macrocytosis accompanied by neutropenia or thrombocytopenia, suggesting that the population of patients with MDS may be higher than estimated. ${ }^{2}$

\section{Clinical features}

MDS constitutes a heterogenous group of hematopoietic stem cell disorders (Table 1) which are characterized by various degrees of anemia, leukopenia, and thrombocytopenia, as well as an increased risk for developing acute myeloid leukemia (AML). Hematopoietic progenitors in MDS show a decreased capacity for differentiation and an increased tendency for apoptosis leading to ineffective hematopoiesis. Cytogenetic and molecular data provide evidence for a clonal hematopoietic stem cell disorder in the majority of cases. Cause, unless associated with prior chemotherapy, radiation, or toxic exposure, eludes discovery. ${ }^{4,5}$ In most cases, ineffective hematopoiesis leads to compensatory bone marrow hyperplasia. Over time many patients develop an increase in bone marrow blasts, about 30\% developing AML.

The clinical variation MDS presents ranges from indolent to life-threatening. Up to $10 \%$ of patients with MDS experience serious bleeding, including hematuria and gastrointestinal, retinal, and central nervous system hemorrhage. They may also develop Sweet's syndrome, splenomegaly (in $10 \%$ to $20 \%$ of patients), and hepatomegaly ( $5 \%$ to $26 \%$ ). In chronic myelomonocytic leukemia, however, the incidence of splenomegaly and hepatomegaly increases to $30 \%$ to $50 \%$, and the spleen may be massively enlarged. In general, lymph node enlargement is seen in 5\% to $15 \%$ of these cases.

To assist in decision making regarding treatment, a riskadapted approach has been adopted and is widely accepted. The International Prognostic Scoring System (IPSS) (Table 2) is based on karyotype, number of significantly cytopenias, and percentage of bone marrow myeloblasts. It provides a tool to measure the probability of survival and risk for progression towards AML in untreated patients with MDS. The IPSS is widely accepted as a valid tool to measure prognosis, and the cytogenetic risk stratification system of the IPSS has proven to be an independent prognostic marker in studies of high-risk MDS and AML following MDS. ${ }^{6}$

According to recommendations of an International Working Group, the major goal of therapy for patients with lower-risk disease (low-risk and intermediate-1 categories, as defined by the IPSS) is hematologic improvement and management of symptoms with the aim of improving the quality of life. ${ }^{?}$

For higher-risk patients (intermediate-2 and high-risk categories), the focus turns to modifying the natural history of the disease and extending survival. ${ }^{8}$ These prognostically distinct therapeutic achievements have only recently been identified as new data demonstrate certain therapies for lower-risk may modify the overall course of the disease improving survival and quality of life. Supportive care for MDS includes red blood cell transfusion, platelet transfusion, iron chelation therapy to reduce transfusional hemosiderosis, and hematologic growth factors (when appropriate) to raise neutrophil and hemoglobin levels. New therapies for this disease, including low-intensity chemotherapies and immunomodulators, may enhance hematologic improvement, decrease the rate of leukemic transformation, and improve overall survival; they are now 
Table I WHO classification and criteria for the myelodysplastic syndromes ${ }^{3}$

\begin{tabular}{|c|c|c|}
\hline Disorder & Peripheral blood & Bone marrow \\
\hline \multirow[t]{3}{*}{ Refractory anemia (RA) } & Anemia & Erythroid dysplasia only \\
\hline & No or rare blasts & $<5 \%$ blasts \\
\hline & & $<15 \%$ ringed sideroblasts \\
\hline \multirow{3}{*}{$\begin{array}{l}\text { Refractory anemia with ringed } \\
\text { sideroblasts (RARS) }\end{array}$} & Anemia & Erythroid dysplasia only \\
\hline & No blasts & $<5 \%$ blasts \\
\hline & & $\geq 15 \%$ ringed sideroblasts \\
\hline \multirow[t]{4}{*}{$\begin{array}{l}\text { Refractory cytopenia with multilineage } \\
\text { dysplasia (RCMD) }\end{array}$} & $\mathrm{Bi}$ - or pan-cytopenia & $\begin{array}{l}\text { Dysplasia in } \geq 10 \% \text { of cells in } 2 \text { or more } \\
\text { myeloid cell lines }\end{array}$ \\
\hline & No or rare blasts & No Auer rods \\
\hline & No Auer rods & $<5 \%$ blasts \\
\hline & Monocytes $<\mathrm{I}, 000 / \mu \mathrm{L}$ & $<15 \%$ ringed sideroblasts \\
\hline \multirow{4}{*}{$\begin{array}{l}\text { Refractory cytopenia with multilineage } \\
\text { dysplasia and ringed sideroblasts } \\
\text { (RCMD-RS) }\end{array}$} & $\mathrm{Bi}$ - or pan-cytopenia & $\begin{array}{l}\text { Dysplasia in } \geq 10 \% \text { of cells in } 2 \text { or more } \\
\text { myeloid cell lines }\end{array}$ \\
\hline & No or rare blasts & No Auer rods \\
\hline & No Auer rods & $<5 \%$ blasts \\
\hline & Monocytes $<\mathrm{I}, 000 / \mu \mathrm{L}$ & $\geq 15 \%$ ringed sideroblasts \\
\hline \multirow{4}{*}{$\begin{array}{l}\text { Refractory anemia with excess blasts-I } \\
\text { (RAEB-I) }\end{array}$} & Cytopenias & Unilineage or multilineage dysplasia \\
\hline & $<5 \%$ blasts & $5 \%$ to $9 \%$ blasts \\
\hline & No Auer rods & No Auer rods \\
\hline & Monocytes $<\mathrm{I}, 000 / \mu \mathrm{L}$ & \\
\hline \multirow{4}{*}{$\begin{array}{l}\text { Refractory anemia with excess blasts- } 2 \\
\text { (RAEB-2) }\end{array}$} & Cytopenias & Unilineage or multilineage dysplasia \\
\hline & $5 \%$ to $19 \%$ blasts & $10 \%$ to $19 \%$ blasts \\
\hline & Auer rods \pm & Auer rods \pm \\
\hline & Monocytes $<\mathrm{I}, 000 / \mu \mathrm{L}$ & \\
\hline \multirow[t]{3}{*}{ MDS-unclassified (MDS-U) } & Cytopenias & $\begin{array}{l}\text { Unilineage dysplasia in granulocytes } \\
\text { or megakaryocytes }\end{array}$ \\
\hline & No or rare blasts & No Auer rods \\
\hline & No Auer rods & $<5 \%$ blasts \\
\hline \multirow[t]{3}{*}{ MDS with del (5q) "5q-syndrome" } & Anemia & $\begin{array}{l}\text { Normal to increased megakaryocytes with } \\
\text { hypolobulated nuclei }\end{array}$ \\
\hline & $<5 \%$ blasts & No Auer rods \\
\hline & $\begin{array}{l}\text { Platelets usually normal } \\
\text { or increased }\end{array}$ & $<5 \%$ blasts Isolated del $(5 q)$ \\
\hline
\end{tabular}

being used to treat low-risk disease. ${ }^{9}$ For patients with high-risk disease, high-intensity treatments such as leukemiatype induction therapy and HSCT are used and are briefly taken into consideration in this review.

As more low-risk patients are being treated with lowintensity chemotherapy and immunomodulating agents, with the aim of altering the trajectory of the disease, the IPSS classification system, which emphasizes pretreatment prognostic factors, becomes more difficult to apply and is becoming an outdated measuring tool. Several investigators have proposed alternative prognostic tools including serial application and incorporating World Health Organization criteria into the IPSS (becoming WPSS), ${ }^{10}$ adding serum lactate dehydrogenase as a prognostic variable, ${ }^{11}$ and more risk stratification of low-risk disease. ${ }^{12}$ New prognostic scoring methods have been proposed by the M.D. Anderson group, taking into account prior lowintensity treatment, secondary disease, performance status, and transfusion requirements, in addition to measurements used in 
Table 2 International Prognostic Scoring System (IPSS) ${ }^{7}$

\begin{tabular}{|c|c|c|c|}
\hline Factor & Value & IPSS score & Notes \\
\hline \multirow[t]{4}{*}{ Blasts } & $5 \%$ or less & 0 & \\
\hline & $5 \%$ to $10 \%$ & 0.5 & \\
\hline & $11 \%$ to $20 \%$ & 1.5 & \\
\hline & $21 \%$ to $30 \%$ & 2.0 & \\
\hline \multirow[t]{3}{*}{ Cytogenetics } & $=$ Good & 0 & Normal; -Y only; 5q- only; or 20q- only \\
\hline & $=$ Intermediate & 0.5 & Abnormalities other than good or poor \\
\hline & $=$ Poor & 1.0 & Complex; 3 or more abnormalities or abnormal chromosome 7 \\
\hline \multirow[t]{2}{*}{ Cytopenias } & $0 / 1$ & 0 & Hemoglobin $<10 \mathrm{~g} / \mathrm{dL}$; absolute neutrophil count $($ ANC) $<\mathrm{I}, 500 / \mu \mathrm{L}$; \\
\hline & $2 / 3$ & 0.5 & platelet count $<100,000 / \mu \mathrm{L} ;$ each count as a value of $\mathrm{I}$ \\
\hline
\end{tabular}

Notes: The numeric scores for the blast \%, the cytogenetic changes and the cytopenias are combined to give the total numeric score. The scores equal a risk category:

- Low.

- Intermediate-I.

- Intermediate-2.

- High.

The risk categories are sometimes combined as

- Low and intermediate-I = low-risk MDS.

- Intermediate-2 and high = high-risk MDS.

the IPSS. ${ }^{13}$ In addition to the biologic and clinical properties of MDS, however, the treatment becomes significantly complicated due to the older population, their co-morbid illnesses, difficulties of access to care, social situations, cognitive status, and the large variation in functional status. Given these factors, decision making about treatment for elderly patients is extremely complex and geriatric factors need to be incorporated into prognostic scoring for MDS.

\section{Quality of life in MDS patients}

When treatments are unsatisfactory there are two main areas to be taken into consideration. In the first, therapeutic approaches which alleviate symptoms but do not affect the underlying disease or prognosis are taken into account. In the second a therapeutic benefit in terms of prognosis is achieved, but a considerable price of toxicity or hospitalization is expected.

Quality of life could be defined as a multidimensional construct based on somatic, emotional, mental, social and behavioral components of well-being and function from the point of view of the patient and/or external observers. ${ }^{14}$

MDS seriously affect the quality of life of patients. Although there have been considerable improvements in available therapies, once diagnosed, the disease will dominate much of the remaining time of the patient and potentially be the cause of death unless competing co-morbidities are present or allogeneic stem cell transplant is possible.

Suffering from MDS causes a substantial and persistent functional decrement in a variety of areas, including the above mentioned, partially due to fatigue ( $81 \%$ of patients). A negative impact on quality of life results from the time and effort required to cope with the disease such as frequent visits to doctors, administering therapy, and managing side effects. Patients have often viewed the emotional impact of MDS (shock, anger, depression, and anxiety) as being more problematic than the physical consequences.

Among the cytopenias which define MDS, anemia has the most prominent influence. Low hemoglobin values are a key determinant of fatigue, a key determinant of quality of life. Although frequent and unusual infections may contribute to physical complaints, this is rarely a major issue in the early stages of the disease. MDS related thrombocytopenic bleeding is usually confined to later stages and progression to leukemia. Moreover, there may be other physiological (rather than psychological) reasons. For other neoplastic diseases, it has been shown that altered cytokine levels contribute to alterations of physical and psychological well-being, ${ }^{15}$ which may be the case in patients with MDS/leukemia; ${ }^{16,17}$ in polytransfused patients the evolving secondary hemochromatosis may also contribute to the number of symptoms MDS patients have to bear. Manifestations may range from increased fatigue to overt heart failure, but due to the underlying disease and the high rate of co-morbidity in the elderly patients, it remains difficult to ascertain the contribution of the various etiologies to the respective symptom. ${ }^{18}$

\section{Management}

\section{Observation ('watch and wait')}

Observation with periodic blood counts, also called 'watch and wait', is generally recommended for a patient 
with a low or intermediate-1 risk hemoglobin level greater than $10 \mathrm{~g} / \mathrm{dL}$ and platelet counts of $50,000 / \mu \mathrm{L}$ to $100,000 / \mu \mathrm{L}$, without need for transfusion. These patients may be able to maintain their usual activity levels without therapy.

Some patients have little change in status for years. However, regular check-ups are important, in that there is a risk of progression to more severe forms of MDS or AML. It is therefore important for a patient to have an MDS specialist do periodic health status evaluations and monitor blood cell counts on a regular basis.

\section{Anemia in MDS: its importance in the geriatric patient}

Recent, extended, observational epidemiologic studies have consistently documented that anemia, even if not severe, is strongly and independently associated with major adverse functional outcomes in community-dwelling of older adults, including the decline in physical and cognitive function, frailty, and disability. ${ }^{19}$ Evidence has also shown that anemia interacts with common co-morbidities to synergistically increase the risk of outcomes such as frailty and mortality. ${ }^{20,21}$ Anemia is most often defined by World Health Organization criteria (hemoglobin $<12 \mathrm{~g} / \mathrm{dL}$ for women and $<13 \mathrm{~g} / \mathrm{dL}$ for men), but these criteria do not consider the debilitating effects of mild anemia on the elderly population. It is not clear if a lower hemoglobin concentration for post-menopausal women is valid or whether the same threshold should be used for both men and women. ${ }^{22}$ Racial differences may be important in assessing optimal hemoglobin concentration, and this research will be important in determining the best supportive care for MDS patients. More research involving the communitydwelling of the elderly population is needed to determine optimal hemoglobin levels for elderly patients and the effect of treatment for mild anemia on outcome. Patients with MDS often have severe anemia, which contributes to their overall prognosis, exacerbating the symptoms of co-morbid illnesses and influencing functional, cognitive, and social deterioration in their lives. Using current prognostic scales, anemia in all MDS patients should be aggressively treated to improve functional and cognitive status in order to improve quality of life.

\section{Anemia in MDS: supportive care strategies} Red blood cell transfusion

Initiation of transfusion therapy should be based on clinical evaluation of anemia-related symptoms and co-morbid illness rather than on a defined hemoglobin level, especially because optimal hemoglobin levels have not been defined for the elderly population. ${ }^{23}$ In clinical practice, pre-transfusion hemoglobin levels in MDS are generally maintained between 8 and $10 \mathrm{~g} / \mathrm{dL}$, but all quality of life studies show significantly greater fatigue and dyspnea in MDS patients rather than in healthy controls, and it is not known whether a more liberal transfusion strategy can improve quality of life or outcome. ${ }^{24}$

\section{Iron chelation therapy}

Chronically transfused MDS patients invariably develop iron overload, which can lead to organ damage and dysfunction. Evidence of the direct role of iron in organ damage in MDS is still limited, and clinical information is usually inferred from other transfusion-dependent anemias, especially thalassemia. The time from onset of transfusion to the development of organ damage caused by iron overload is well defined in the thalassemia cohort, but this time may not apply to the elderly MDS patient.

There is evidence from a small-scale study that iron chelation has efficacy in elderly MDS patients. The administration of oral deferasirox (Exjade ${ }^{\circledR}: 20$ to $40 \mathrm{mg} / \mathrm{kg}$ per day) in transfusion-dependent MDS patients induces a significant decrement of the mean serum ferritin after 12 months of therapy. The labile plasma iron concentration normalized in all patients within 3 months and was sustained throughout the first year of treatment. Reversible elevation in serum creatinine occurred in $25 \%$ of the patients. ${ }^{25}$

Moreover, in patients who received standard chelation therapy with subcutaneous deferoxamine (Desferal ${ }^{\circledR}$ ), deferiprone $\left(\right.$ Ferriprox $\left.^{\circledR}\right)$, or deferasirox, or low-dose chelation with subcutaneous bolus deferoxamine or intravenous deferoxamine after transfusions the median overall survival from the time of diagnosis was better than in nonchelated patients (115 months vs 51 months; $P<0.0001)$. After stratification by prognostic variables including IPSS category, age, sex, and transfusion requirements, the survival difference favoring the chelated group remained significant. ${ }^{26}$

Although these studies suggest that iron chelation treatment is beneficial, there are still uncertainties. More research needs to be done to determine the best time to initiate treatment. Oral iron chelation therapy is expensive and is linked to renal insufficiency. This therapy may be difficult for older patients to tolerate. More intense studies are needed to determine the survival benefit, cost feasibility, and the right moment to begin the therapy. 


\section{Erythropoiesis-stimulating agents}

Recombinant human erythropoietins in doses ranging from 30,000 to 60,000 units/week can be effective in MDS. A meta-analysis of more than 200 patients from 17 studies showed that treatment rHuEpo as monotherapy induced erythroid responses in about $15 \%$ of patients with MDS. ${ }^{27}$ Two phase 3, randomized controlled trials evaluated the use of rHuEpo vs placebo. The larger of these studies enrolled 87 patients with hemoglobin less than $9 \mathrm{~g} / \mathrm{dL}$ and bone marrow blasts less than $10 \%$. The response rate was $37 \%$. Patients with refractory anemia (RA) in the FrenchAmerican-British (FAB) classification system had a higher probability of response, but in patients with refractory anemia with ringed sideroblasts (RAS) and refractory anemia with excess blasts (RAEB), the responses were not significantly different from those with placebo. A higher response rate was observed in patients without prior transfusions and in those with low pretreatment erythropoietin levels $(<150$ to $200 \mathrm{U} / \mathrm{L}){ }^{28}$

The most widely adopted response criteria are those of the Nordic and French MDS Groups, which define a complete erythroid response as an increase in hemoglobin level to at least $11.5 \mathrm{~g} / \mathrm{dL}$ without red blood cell transfusions; a partial erythroid response is defined as no transfusion requirement or an increased hemoglobin level of more than $1.5 \mathrm{~g} / \mathrm{dL}$ for patients with non-transfused anemia. ${ }^{29}$ There is no consistent information on the length of treatment before assessing response. Most often, responses occur between 12 and 16 weeks, but additional responses may be observed if treatment is continued as long as 6 months. In a phase II trial in 281 MDS patients conducted for The Greek MDS Study Group Terpos et al prolonged administration of rHuEpo produces high and long-lasting erythroid response rete in MDS patients with low blast counts, particularly in those with pretreatment serum Epo levels of $<150 \mathrm{U} / \mathrm{L}$ and good cytogenetic prognosis. ${ }^{30}$ They evaluated the response to treatment after 12 and 26 weeks of therapy with a significant increase in response rate observed at week 26 in RA, RARS and RAEB-I patients, as the response probability increased with treatment duration.

Treatment with darbepoetin alpha, a highly glycosylated erythropoiesis-stimulating agent with a longer half-life than epoetin alpha, has produced comparable response rates at dosing intervals ranging from 1 to 3 weeks. ${ }^{31}$ Treatment with erythropoiesis-stimulating agents may improve quality of life in patients and reduce the need for transfusion support.

Golshayan et al conducted a pooled analysis in 1,869 MDS patients with low-risk disease according to treatment with erythropoiesis-stimulating agents. ${ }^{32}$ A survival benefit was observed in patients who received an erythropoiesisstimulating agent, and this difference was maintained even after outcomes were corrected for FAB type, baseline transfusion requirements, IPSS category, prior treatments, and interval from diagnosis.

Treatment with erythropoiesis-stimulating agents has come under scrutiny during the past year. The US Food and Drug Administration (FDA) has strengthened safety warnings based on concerns in solid-tumor trials showing increased mortality from the underlying disease and increased risk for thromboembolism, particularly in studies targeting higher hemoglobin levels $(>12 \mathrm{mg} / \mathrm{dL})$. This information prompted the Centers for Medicare and Medicaid Services to limit coverage for erythropoiesis-stimulating agents for the treatment of chemotherapy-induced anemia, but they have elected to exclude MDS from the national coverage decision. Available data involving the use of erythropoiesis-stimulating agents in MDS show that these agents improve symptoms and decrement transfusion requirements. The extent to which they improve functional and cognitive symptoms associated with anemia in MDS patients has not been documented and should be a focus for further research.

\section{Treatment of other cytopenias}

\section{Neutropenia and secondary infections}

Severe neutropenia in low-risk MDS usually occurs in the setting of pancytopenia, but can be an isolated clinical problem. There is no indication for prophylactic use of granulocyte colony-stimulating factor (G-CSF) in MDS, but this drug can be an option for individual patients with severe neutropenia and recurrent infections. Prophylactic antibiotics are also used in MDS patients with neutropenia. Many studies document the efficacy of using prophylactic fluoroquinolone antibiotics in neutropenic patients to prevent febrile neutropenia and bacteremia and to reduce days of hospitalization. ${ }^{33}$

Prophylaxis in elderly MDS patients has drawbacks that need to be considered. Numerous reports have linked the use of prophylactic antibiotics to the emergence of resistant organisms, an important consideration for patients who may be expected to have neutropenia for a long period.

Where there are high levels of resistance to fluoroquinolones (such as in some nursing homes and assisted living communities), the resistance pattern should prompt a reconsideration of prophylactic strategies. It is also important to ensure that prophylactic antibiotics and antifungals do 
not interact with other medications in patients using large numbers of prescription drugs to treat co-morbid illnesses. These patients may also need assistance to administer medications.

\section{Thrombocytopenia and bleeding-related disorders}

Severe thrombocytopenia with chronic bleeding is a serious clinical problem in MDS and is associated with shortened survival and impaired quality of life. Platelet transfusions may provide short-term relief for bleeding tendencies but cannot prevent long-term symptoms.

Megakaryocytopoiesis has been difficult to stimulate in vivo. The FDA recently approved a drug for treatment of immune thrombocytopenia, namely AMG 531. This drug is currently in clinical trial for MDS patients and may prove beneficial to elderly patients with this disease. ${ }^{34}$

\section{Treatment strategies Curative therapy for MDS}

High-dose chemotherapy followed by HSCT is presently the only treatment with curative potential in MDS. Historically, experience has demonstrated that about $40 \%$ of patients may be cured with hematopoietic stem cell transplant, but advanced age, co-morbid medical problems, procedure morbidity and mortality, and lack of donor availability limit the procedure to a small, select minority. ${ }^{35,36}$ In particular subjects older than 50 years, age and blast percentage at HSCT are the most important factors for outcome of trasplanted patients ${ }^{37,38}$ and for the choice of myeloablative or non-myeloablative conditioning regimen. ${ }^{39}$ Moreover, due to increasing age that non-myeloablative transplant will be done, the availability of transplant for elderly MDS is expanding.

Decision models are available to assist in determining which MDS patients should undergo hematopoietic stem cell transplant and whether they are fit. ${ }^{40,41}$ However, these models do not consider functional, cognitive, and social situations that affect elderly patients' long-term ability to lead independent, functional lives and to maintain an acceptable quality of life after transplantation. Optimal models for selection of older patients for aggressive therapy with hematopoietic stem cell transplant have not been developed.

\section{Non-curative therapy for MDS}

Growth factors can be an effective tool in treating low risk MDS, but the vast majority of MDS patients with transfusion requirements of two units of red blood cells per month or elevated erythropoietin levels are unlikely to respond to this treatment.
In the past decades many attempts to improve survival of MDS patients have been made, but the results have always been disappointing.

\section{Hormonal therapy}

Hormone therapy has been used to improve hematologic parameters in MDS patients. Androgens have been suggested to improve anemia, leukopenia, and thrombocytopenia, but results from trials have been inconsistent. ${ }^{42}$

\section{Differentiating agents}

Low-dose cytarabine has been reported to possess the ability to induce differentiation of the leukemic cell line in vitro, and trials have been done on patients with MDS. ${ }^{43-45}$ Retinoid differentiating activity has been established in studies of promyelocytic leukemia, but trials in MDS, however, have failed to show any impact. ${ }^{46}$ Retinoic acid may also accelerate the transformation of MDS to AML, ${ }^{47}$ but again studies on the treatment of MDS patients with other differentiating agents such as vitamin D3, interferon alpha, and interferon gamma are quite disappointing. ${ }^{48}$

\section{Chemotherapy}

Chemotherapy trials for MDS using standard antileukemic drugs in different dosages and combinations using daunorubicin, cytosine arabinoside (ara-C), 6-thioguanine indicate that CRs have occurred ( $8 \%$ to $56 \%$ ) but at the cost of a high incidence of deaths from toxicity (24\% to $64 \%) .{ }^{48}$

While the majority of MDS and MDS/AML patients have hypercellular bone marrow, $10 \%$ to $15 \%$ have marrow hypocellularity. Hypocellular MDS patients may have longer survival and may progress to AML less frequently. These hypocellular MDS/AML patients represent the best candidates to observation or growth factors administration also because with chemotherapy they may have more complications and less chance of achieving remission. ${ }^{49}$

\section{New strategies}

There is evidence that treating patients with immunomodulatory therapy or low-intensity therapy may alter the longterm course of the disease and improve quality of life and survival.

\section{Lenalidomide $\left(\right.$ Revlimid $\left.^{\circledR}\right)$}

The pathogenesis of MDS involves a complex biologic interaction that fosters increased angiogenesis, overproduction of proinflammatory cytokines, accelerated apoptosis of hematopoietic progenitors, and autocrine stimulation by vascular endothelial growth factor. Immunomodulatory drugs 
such as lenalidomide can alter the bone marrow environment and can potentially interfere with this complex series of events. Lenalidomide is a second-generation immunomodulatory drug; it is more potent and less neurotoxic than its predecessor, thalidomide. The MDS-003 clinical trial was the pivotal study evaluating the efficacy of lenalidomide in lowerrisk, transfusion-dependent patients with a chromosome 5q31 deletion, with or without other cytogenetic abnormalities. The clinical trial showed that $76 \%$ of patients who received lenalidomide had a $50 \%$ or greater reduction in transfusion requirements; $67 \%$ became transfusion-independent, with a rise in hemoglobin of $1 \mathrm{~g} / \mathrm{dL}$ or more. The median duration of transfusion independence was 2 years. The most common adverse events were neutropenia and thrombocytopenia. ${ }^{50}$

Lenalidomide has also been evaluated in low-risk patients without del(5q). In a large, multicenter trial, 56 of 214 patients achieved red blood cell transfusion independence with a rise in hemoglobin of $1 \mathrm{~g} / \mathrm{dL}$ or more, and an additional $17 \%$ had a $50 \%$ or greater reduction in transfusion requirements. ${ }^{51}$

An advantage of using this agent to treat elderly patients with MDS is that it is an oral agent that can be easily managed in the outpatient setting. Patients need careful monitoring of blood counts and supportive transfusion, but they do not need to come to the physician's office to receive infusional therapy and do not need any inpatient monitoring.

\section{Methyltransferase inhibitors}

Hypermethylation of cytoside residues on DNA and consequent chromatin condensation are key features of the malignant phenotype that lead to epigenetic silencing of genes involved in the control of normal cell growth and differentiation. Methyltransferase inhibitors are a class of medications that deplete nuclear DNA methyltransferase, the enzyme responsible for methylation of the DNA; as a consequence, they promote demethylation in newly synthesized DNA.

Two Methyltransferase inhibitors are approved for treatment of MDS: azacitidine and decitabine. Both are cytosine analogues that are distinguished by their sugar moieties, which may account for different activity.

\section{Azacitidine (Vidaza $\left.{ }^{\circledR}\right)$}

Encouraging results from the phase 2 trials of azacitidine, initiated by the Cancer and Leukemia Group B, led to a phase 3 study that compared treatment with azacitidine vs best supportive care in patients with MDS (any FAB subtype). Patients in the best supportive care arm were allowed to cross over to azacitidine if they experienced progression of disease.
Responses to azacitidine were observed in all FAB subtypes, with a complete response (CR) plus partial response (PR) in $22 \%$ and hematologic improvement in $37 \%$ of patients. Median time to AML transformation was 13 months in the best supportive care group compared with 21 months in the azacitidine $\operatorname{arm}(P=0.007) .{ }^{52}$

A phase 3, randomized, multicenter trial (AZA-001) compared azacitidine treatment using the FDA-approved dosing schedule with conventional care regimens in previously untreated patients having IPSS intermediate-2 or high-risk RAEB, RAEB in transformation (RAEB-t), or chronic myelomonocytic leukemia. ${ }^{53}$ Before randomization, patients selected three Conventional Care Regimens strategies: a) best supportive care; b) low-dose cytarabine; c) standard AML-type induction therapy with consolidation chemotherapy. Azacitidine was continued until evidence of disease progression or treatment intolerance. In this study, 358 patients were randomized to either azacitidine $(n=179)$ or conventional care regimens $(n=179)$ with a primary endpoint of overall survival. Azacitidine was administered for a median of nine cycles. There was a significantly improvement in overall survival (median, 24.4 months vs 15 months with conventional care regimens; $P=0.0001$, hazard ratio $=0.058$ ) across all IPSS categories. Survival after 2 years was nearly doubled with azacitidine (51\% vs $26 \%, P<0.0001)$. Azacitidine treatment was superior to all conventional care regimens, with corresponding extension in median overall survival of 12.9 months vs best supportive care, 9.1 months vs low-dose cytarabine, and 8.7 months vs standard induction therapy. Response rates also favored azacitidine; more patients achieved major and minor responses with azacitidine than with any conventional care regimens. ${ }^{53}$ The improvement in overall survival despite a modest CR rate of $17 \%$ indicates that azacitidine acts in a distinctive way that differs from that of conventional cytotoxic drugs and leads to reduced leukemic potential.

\section{Decitabine $\left(\right.$ Dacogen $\left.^{\circledR}\right)$}

A phase 3 trial of another MTI, decitabine, led to FDA approval for this drug for the treatment of AML and MDS (intermediate-1, intermediate-2, and high-risk IPSS). Patients were randomized to receive best supportive care or decitabine $\left(15 \mathrm{mg} / \mathrm{m}^{2}\right)$, given intravenously over 3 hours every 8 hours for 3 days and repeated every 6 weeks for up to 6 cycles. Patients who received best supportive care could cross over and receive decitabine after transformation to AML. End points for the trial were $\mathrm{CR}$ and PR, time into AML transformation, or death. The overall response rate was 
$30 \%$ (CR 9\%, PR 8\%, hematologic improvement 13\%), but there was no difference between treatment arms in the median time to death or transformation into AML. Overall mortality in patients treated with decitabine was $10 \%$; neutropenia developed in $87 \%$ of these patients and grade 3 or 4 thrombocytopenia occurred in $85 \% .{ }^{54}$

Apparently the two phase III trials on methyltransferase inhibitors have different results, but they cannot be correctly compared because of the different design of the 2 studies. In fact, the control harm are quite different: in the study of azacitidine the control harm is composed by best supportive care, low-dose cytarabine, or intensive chemotherapy as selected by investigators before randomization, while in the decitabine study the control harm is only best supportive care. However, the comparison of results obtained in decitabine trial with the group received only best supportive care in the control harm of azacitidine study gave similar results.

\section{Antithymocyte globulin}

\section{(ATG; Thymoglobuline ${ }^{\circledR} ;$ Atgam $^{\circledR}$ )}

Some MDS patients have disease characterized by lymphocytes that destroy precursors of normal red cells, neutrophils and platelets. ATG is an immune globulin that is obtained from rabbits or horses and is given intravenously, destroys lymphocytes and improves blood counts in some MDS patients. A single course of ATG restored hemopoiesis in some patients with MDS, particularly in patients with RA and RAEB with a median response duration of 10 months and is well tolerated with only immediate fever and chills as common side effects after ATG administration. ${ }^{55}$

It is possible to identify patients with better or worse chances of responding to ATG or other immunosuppressive agents such as cyclosporine..$^{56-58}$ These are those with low or intermediate-1 IPSS risk or that express HLA-DR15 antigen.

\section{Current clinical trials in MDS}

The goal of clinical trials for MDS is to improve treatment and quality of life and to increase survival. Patients' participation in clinical trials is needed to improve standard therapies, so that all MDS patients can one day be cured of this disease. Some clinical trials are for all MDS-risk types; others are for either lower-risk or higher-risk MDS. Patients should speak to their doctors about the benefits of treatment in a specific clinical trial. Eligibility for a trial may depend on the patient's age, risk type and previous treatment for MDS.

There are several clinical trials to study treatment with combinations of FDA-approved drugs, such as azacitidine or decitabine, and AML-type chemotherapy. The idea of combining agents is that, since each works in different ways to kill cancer cells, using them together may kill more MDS cells or be as effective as standard MDS therapies, but with less-toxic side effects.

Trials are currently estimating: i) the effectiveness of arsenic trioxide $\left(\right.$ Trisenox ${ }^{\circledR}$ ) in combination with azacitidine or with tipifarnib $\left(\right.$ Zarnestra $\left.^{\circledR}\right)$, a farnesyl transferase inhibitor, and gemtuzumab ozogamicin (Mylotarg ${ }^{\circledR}$ ); ii) if the duration of response improves with azacitidine maintenance for patients who achieve a complete or partial remission after intensive chemotherapy; iii) the effectiveness of clofarabine $\left(\right.$ Clolar $\left.^{\circledR}\right)$ in combination with AML-type chemotherapy; iv) the effect of lonafarnib (Sarasar ${ }^{\circledR}$ ) and tipifarnib (Zarnestra $^{\circledR}$ ) on transfusion independence for patients who receive between 1 to 8 platelet transfusions every 4 weeks; v) the effectiveness of valproic acid (Depakene ${ }^{\circledR}$ ) in combination with decitabine; vi) the effectiveness of vorinostat $\left(Z_{\text {olinza }}{ }^{\circledR}\right)$ in combination with azacitidine.

\section{Conclusions}

There are many complex variables in the treatment of elderly patients with MDS. As the elderly population increases worldwide, the proportion of elderly patients with MDS will also rise and the costs of treating this disease will be substantial. Evidence-based strategies are needed to determine which patients are best suited to low-intensity treatment, high-intensity treatment with hematopoietic stem cell transplant, best supportive care, or no treatment. These stratification methods should consider biologic factors of the disease, co-morbid illness, depression, functional status, cognitive status, and social situation.

To make a choice of management in older MDS patients the first step must be an integrating geriatric and oncology nursing strategies allows an individualized approach toward this unique population. All the factors influence the potential inability of the older patient to tolerate certain intensive forms of therapy must be considered and particularly multiple medical problems affect older population and use medications to manage them. Particularly important in the elderly MDS patient is the evaluation of the age-related decline in normal bone marrow function, including diminished capacity for response to stressors such as infection or myelosuppressive treatments. Further observation may be useful to determine if the patient has an indolent or progressive course, to evaluate if the MDS is presented as isolated but persistent mild anemia or as a more progressive and aggressive anemia state that accumulates excess blasts in the marrow and leads to fatal AML. So, patients may be 
categorized by age, co-morbidities and by stable vs unstable disease before suggesting specific treatment approaches. To improve access to treatment, emphasis must be placed on oral therapies, which can be easily administered in the outpatient setting with growth-factor support to minimize transfusion. Intensive efforts are needed to find strategies that keep elderly patients functional and in their homes during treatment, minimizing time in the physician's office and hospital.

In conclusion, careful evaluation of functional status, ability to tolerate treatments, effect of disease progression, and general overall health can provide the best opportunity for support of older patients, considering that palliation and supportive care represent important components to maximizing quality of life.

\section{Disclosures}

The authors declare no conflict of interest.

\section{Acknowledgments}

This work was supported by Associazione Italiana per la Ricerca sul Cancro (AIRC), Milan; the Ministry of Education, Universities and Research (MIUR, PRIN Projects 2008); the Ministry of Health, Progetto Oncologia 2006, Humanitas Mirasole S.p.A., Rome, Italy.

The authors would like to thank Prof C Perillo for English editing of the manuscript.

\section{References}

1. Rollison DE, Howlader N, Smith MT, et al. Epidemiology of myelodysplastic syndromes and chronic myeloproliferative disorders in the United States 2001-2004, using data from the NAACCR and SEER programs. Blood. 2008;112:45-52.

2. Guralnik JM, Eisenstaedt RS, Ferrucci L, et al. Prevalence of anemia in persons 65 years and older in the United States: evidence for a high rate of unexplained anemia. Blood. 2004;104:2263-2268.

3. Howe RB, Porwit-MacDonald A, Wanat R, et al. The WHO classification of MDS does make a difference. Blood. 2004;103:3265-3270.

4. Alessandrino EP, Amadori S, Cazzola M, et al. Myelodysplastic syndromes: recent advances. Haematologica. 2001;86:1124-1157.

5. Hellström-Lindberg E, Willman C, Barrett J, Saunthararajah Y. Achievements in understanding and treatment of myelodysplastic syndromes. American Society of Hematology Education Program Book. 2000:110-132.

6. De Witte T, Suciu S, Verhoef G, et al. Intensive chemotherapy followed by allogeneic or autologous stem cell transplantation for patients with myelodysplastic syndromes (MDSs) and acute myeloid leukemia following MDS. Blood. 2001;98:2326-2331.

7. Greenberg P, Cox C, LeBeau MM, et al. International scoring system for evaluating prognosis in myelodysplastic syndromes. Blood. 1997;89:2079-2088.

8. Cheson BD, Bennett JM, Kantarjian H, et al. Report of an international working group to standardize response criteria for myelodysplastic syndromes. Blood. 2000;96:3671-3674.

9. Adès L, Boehrer S, Prebet T, et al. Efficacy and safety of lenalidomide in intermediate- 2 or high-risk myelodysplastic syndromes with $5 \mathrm{q}$ deletion: results of a phase 2 study. Blood. 2009;113:3947-3952.
10. Malcovati L, Germing U, Kuendgen A, et al. Time-dependent prognostic scoring system for predicting survival and leukemic evolution in myelodysplastic syndromes. J Clin Oncol. 2007;25:3503-3510.

11. Germing U, Hildebrandt B, Pfeilstöcker M, et al. Refinement of the international prognostic scoring system (IPSS) by including LDH as an additional prognostic variable to improve risk assessment in patients with primary myelodysplastic syndromes (MDS). Leukemia. 2005;19:2223-2231.

12. Garcia-Manero G, Shan J, Faderl S, et al. A prognostic score for patients with lower risk myelodysplastic syndrome. Leukemia. 2008;22:538-543.

13. Kantarjian H, O'Brien S, Ravandi F, et al. Proposal for a new risk model in myelodysplastic syndrome that accounts for events not considered in the original International Prognostic Scoring System. Cancer. 2008;113:1351-1361

14. Bullinger M, Hasford J. Evaluating quality-of-life measures for clinical trials in Germany. Control Clin Trials. 1991;12:91S-105S.

15. Rich T, Innominato PF, Boerner J, et al. Elevated serum cytokines correlated with altered behavior, serum cortisol rhythm, and dampened 24-hour rest-activity patterns in patients with metastatic colorectal cancer. Clin Cancer Res. 2005;11:1757-1764.

16. Meyers CA, Albitar M, Estey E. Cognitive impairment, fatigue, and cytokine levels in patients with acute myelogenous leukemia or myelodysplastic syndrome. Cancer. 2005;104:788-793.

17. Hsu HC, Lee YM, Tsai WH, et al. Circulating levels of thrombopoietic and inflammatory cytokines in patients with acute myeloblastic leukemia and myelodysplastic syndrome. Oncology. 2002;63:64-69.

18. Tefferi A. Iron chelation therapy for myelodysplastic syndrome: If and when. Mayo Clin Proc. 2006;81:197-198.

19. Chaves PH: Functional outcomes of anemia in older adults. Semin Hematol. 2008;45:255-260.

20. Fried LP, Tangen CM, Walston J, et al. Frailty in older adults: evidence for a phenotype. J Gerontol A Biol Sci Med Sci. 2001;56:M146-M156.

21. Zakai NA, Katz R, Hirsch C, et al. A prospective study of anemia status, hemoglobin concentration, and mortality in an elderly cohort: the Cardiovascular Health Study. Arch Intern Med. 2005;165:2214-2220.

22. Chaves PH, Xue QL, Guralnik JM, et al. What constitutes normal hemoglobin concentration in community-dwelling disabled older women? J Am Geriatr Soc. 2004;52:1811-1816.

23. Bowen D, Culligan D, Jowitt S, et al. Guidelines for the diagnosis and therapy of adult myelodysplastic syndromes. Br J Haematol. 2003;120:187-200.

24. Jansen AJ, Essink-Bot ML, Beckers EA, et al. Quality of life measurement in patients with transfusion-dependent myelodysplastic syndromes. Br J Haematol. 2003;121:270-274.

25. List AF, Baer MR, Steensma D, et al. Deferasirox (ICL670; Exjade) reduces serum ferritin (SF) and labile plasma iron (LPI) in patients with myelodysplastic syndrome [abstract 1470]. Blood (ASH Annual Meeting Abstracts). 2007;110:440a.

26. Rose C, Brechignac S, Vassilief D, et al. Positive impact of iron chelation therapy (CT) on survival in regularly transfused MDS patients; a prospective analysis by the GFM [abstract 249]. Blood (ASH Annual Meeting Abstracts). 2007;110:80a.

27. Hellström-Lindberg E: Efficacy of erythropoietin in the myelodysplastic syndromes: a meta-analysis of 205 patients from 17 studies. Br J Haematol. 1995;89:67-71.

28. A randomized double-blind placebo-controlled study with subcutaneous recombinant human erythropoietin in patients with low-risk myelodysplastic syndromes. Italian Cooperative Study Group for rHuEpo in Myelodysplastic Syndromes. Br J Haematol. 1998;103:1070-1074.

29. Hellström-Lindberg E, Negrin R, Stein R, et al. Erythroid response to treatment with G-CSF plus erythropoietin for the anaemia of patients with myelodysplastic syndromes: proposal for a predictive model. Br J Haematol. 1997;99:344-351.

30. Terpos E, Mougiou A, Kouraklis A, et al. Prolonged administration of erythropoietin increases erythroid response rate in myelodysplastic syndromes: a phase II trial in 281 patients. $\mathrm{Br} J$ Haematol. 2002;118:174-180. 
31. Giraldo P, Nomdedeu B, Loscertales J, et al. Darbepoetin alpha for the treatment of anemia in patients with myelodysplastic syndromes. Cancer. 2006;107:2807-2816.

32. Golshayan AR, Jin T, Maciejewski J, et al. Efficacy of growth factors compared to other therapies for low-risk myelodysplastic syndromes. Br J Haematol. 2007;137:125-132.

33. NCCN practice guidelines for the myelodysplastic syndromes. National Comprehensive Cancer Network. Oncology. 1998;12:53-80.

34. Tiu RV, Sekeres MA. The role of AMG-531 in the treatment of thrombocytopenia in idiopathic thrombocytopenic purpura and myelodysplastic syndromes. Expert Opin Biol Ther. 2008;8:1021-1030.

35. Galili N, Cerny J, Raza A. Current treatment options: impact of cytogenetics on the course of myelodysplasia. Curr Treat Options Oncol. 2007;8:117-128.

36. De Witte T, Zwaan F, Hermans J, et al. Allogeneic bone marrow transplantation for secondary leukemia and myelodysplastic syndrome: a survey by the Leukaemia Working Party of the European Bone Marrow Transplantation Group (EBMTG). Br J Haematol. 1990;74:151-155.

37. Kuendgen A, Strupp C, Aivado M, et al. Myelodysplastic syndromes in patients younger than age 50. J Clin Oncol. 2006;24:5358-5365.

38. Sierra J, Pérez WS, Rozman C, et al. Bone marrow transplantation from HLA-identical sibvling as treatment for myelodysplasia. Blood. 2002;100:1997-2004.

39. Warlick ED, Cioc A, Defor T, et al. Allogeneic stem cell transplantation for adults with myelodysplastic syndromes: importance of pretransplant disease burden. Biol Blood Marrow Transplant. 2009;15:30-38.

40. Appelbaum FR, Anderson J. Allogeneic bone marrow transplantation for myelodysplastic syndrome: outcomes analysis according to IPSS score. Leukemia. 1998;12(Supp1 1):S25-S29.

41. Cutler CS, Lee SJ, Greenberg P, et al. A decision analysis of allogeneic bone marrow transplantation for the myelodysplastic syndromes: delayed transplantation for low-risk myelodysplasia is associated with improved outcome. Blood. 2004;104:579-585.

42. Baccarani M, Tura S. Differentiation of myeloid leukaemic cells: new possibilities for therapy. Br J Haematol. 1979;42:485-487.

43. Wisch JS, Griffin JD, Kufe DW. Response of preleukemic syndromes to continuous infusion of low-dose cytarabine. N Engl J Med. 1983;309: 1599-1602.

44. Miller KB, Kim K, Morrison FS, et al. The evaluation of low-dose cytarabine in the treatment of myelodysplastic syndromes: a phase-III intergroup study. Ann Hematol. 1992;65:162-168.

45. Clark RE, Ismail SA, Jacobs A, et al. A randomized trial of 13-cis retinoic acid with or without cytosine arabinoside in patients with the myelodysplastic syndrome. Br J Haematol. 1987;66:77-83.
46. Garewal H, Greenberg P, List A, et al. N-(4-hydroxyphenyl) retinamide [4-HPR] therapy in myelodysplastic syndromes (MDS): possible disease acceleration by retinoids. Blood (ASH Annual Meeting Abstracts). 1987;70:228a.

47. Cheson BD. The myelodysplastic syndromes: current approaches to therapy. Ann Intern Med. 1990;112:932-941.

48. Saba HI, Shamim F, Ismail R, et al. Acute leukemic transformation in myelodysplastic syndrome. [abstract]. Blood (ASH Annual Meeting Abstracts). 1992;80:459a.

49. Gafter-Gvili A, Fraser A, Paul M, Leibovici L. Meta-analysis: antibiotic prophylaxis reduces mortality in neutropenic patients. Ann Intern Med. 2005;142:979-995.

50. List A, Dewald G, Bennett J, et al. Lenalidomide in the myelodysplastic syndrome with chromosome 5q deletion. $N$ Engl J Med. 2006;355: 1456-1465.

51. Raza A, Reeves JA, Feldman EJ, et al. Phase 2 study of lenalidomide in transfusion-dependent, low-risk, and intermediate-1 risk myelodysplastic syndromes with karyotypes other than deletion 5q. Blood. 2008;111:86-93.

52. Silverman LR, Demakos EP, Peterson BL, et al. Randomized controlled trial of azacitidine in patients with the myelodysplastic syndrome: a study of the cancer and leukemia group B. J Clin Oncol. 2002;20: 2429-2440.

53. Fenaux P, Santini V, Hellstrom-Lindberg E, et al. Efficacy of azacitidine compared with that of conventional care regimens in the treatment of higher-risk myelodysplastic syndromes: a randomised, open-label, phase III study. Lancet Oncol. 2009;10:223-232.

54. Kantarjian H, Issa JP, Rosenfeld CS, et al. Decitabine improves patient outcomes in myelodysplastic syndromes: results of a phase III randomized study. Cancer. 2006;106:1794-1803.

55. Molldrem JJ, Caples M, Mavroudis D, et al. Antithymocyte globulin for patients with myelodysplastic syndrome. Br J Haematol. 1997;99: 699-705.

56. Sorror ML, Sandmaier BM, Storer BE, et al. Comorbidity and disease status based risk stratification of outcomes among patients with acute myeloid leukemia or myelodysplasia receiving allogeneic hematopoietic cell transplantation. J Clin Oncol. 2007;25:4246-4254.

57. Sloand EM, Wu CO, Greenberg P, et al. Factors affecting response and survival in patients with myelodysplasia treated with immunosuppressive therapy. J Clin Oncol. 2008;26:2505-2511.

58. Saunthararajah Y, Nakamura R, Nam JM, et al. HLA-DR15 (DR2) is ovvererepresented in myelodysplastic syndrome and aplastic anemia and predicts a response to immunosuppression in myelodysplastic syndrome. Blood. 2002;100:1570-1574.
Clinical Interventions in Aging

\section{Publish your work in this journal}

Clinical Interventions in Aging is an international, peer-reviewed journal focusing on evidence-based reports on the value or lack thereof of treatments intended to prevent or delay the onset of maladaptive correlates of aging in human beings. This journal is indexed on PubMed Central, MedLine, the American Chemical Society's 'Chemical

\section{Dovepress}

Abstracts Service' (CAS), Scopus and the Elsevier Bibliographic databases. The manuscript management system is completely online and includes a very quick and fair peer-review system, which is all easy to use. Visit http://www.dovepress.com/testimonials.php to read real quotes from published authors. 